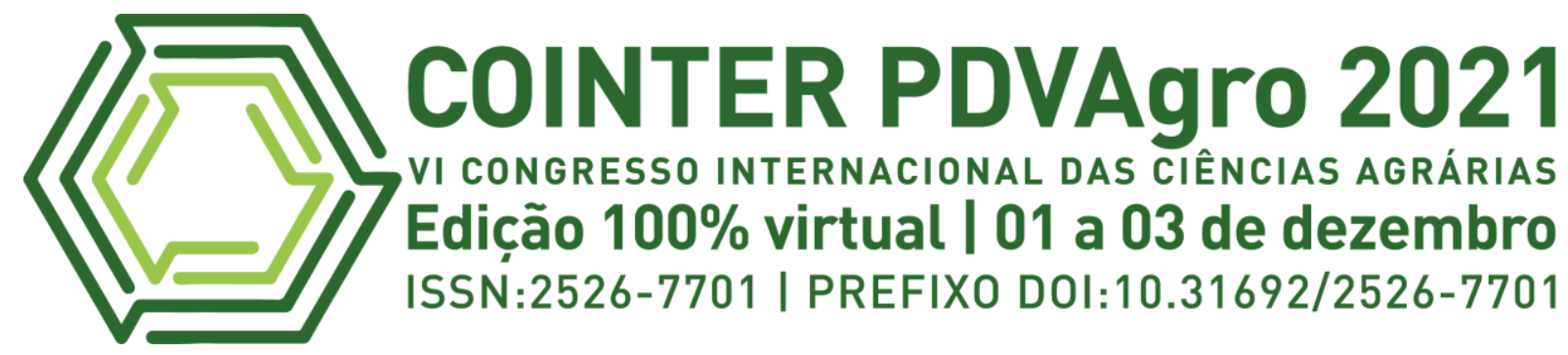

\title{
ANÁLISE DA ADEQUAÇÃO DOS RÓTULOS DE MARGARINAS À LEGISLAÇÃO BRASILEIRA
}

\section{ANÁLISIS DE LA ADECUACIÓN DE LAS ETIQUETAS DE LAS MARGARINAS A LA LEGISLACIÓN BRASILEÑA}

\section{ANALYSIS OF THE ADEQUACY OF MARGARINE LABELS TO BRAZILIAN LEGISLATION}

\author{
Apresentação: Comunicação Oral
}

Carén Beatriz dos Santos Felix da Silva ${ }^{1}$; Andréa Renilda Silva Soares ${ }^{2}$; Mauricio da Silva Souza ${ }^{3}$; Adalberto Francisco da Silva Júnior ${ }^{4}$; Moacyr Cunha Filho ${ }^{5}$

\section{RESUMO}

DOI: https://doi.org/10.31692/2526-7701.VICOINTERPDVAgro.0218

A rotulagem de alimentos concedem a comunicação entre empreendimentos e consumidores e possibilita, ao segundo grupo, conhecer e eleger os produtos que farão parte da sua alimentação. Neste sentido, o presente estudo teve o intuito de avaliar rótulos de diferentes marcas de margarinas, comercializadas na cidade do Recife-PE e confrontando-os com as legislações. Foram coletadas 11 marcas de margarinas aleatoriamente e realizada a análise de suas rotulagens. Para a identificação das embalagens avaliadas, foram atribuídas letras consecutivas e arbitrariamente. As análises foram realizadas de acordo com as informações estabelecidas pelas RDC n ${ }^{\circ}$ 359/03 (BRASIL, 2003a), 360/03 (BRASIL, 2003b) e 259/02 (BRASIL, 2002). Os resultados puderam demonstrar que exceto as marca A, D e K, $100 \%$ das marcas em todos os atributos estão dentro dos parâmetros estabelecidos pela legislação brasileira através da Resolução RDC no 259 de 20 de setembro de 2002 (BRASIL, 2002). Porém as 3 marcas citadas acima não apresentaram informações em sua rotulagem quanto a ausência de glúten. Sob as informações nutricionais, segundo a Resolução n 359 e 360 de 2003 que com exceção da marca B, H e I no que diz respeito ao teor de fibra alimentar $100 \%$ das marcas estudadas apresentam as informações exigidas pela legislação. No que diz respeito aos teores de cálcio, ferro e colesterol identificou-se respetivamente valores de $100 \%$ das amostras não apresentam os teores. Conclui-se que foi identificado irregularidades nos critérios não nutricionais, devido algumas das marcas não apresentarem informação sob ausência de glúten.

Palavras-Chave: Adequabilidade, comunicação, informações, verificação.

\section{RESUMEN}

El etiquetado de los alimentos garantiza la comunicación entre las empresas y los consumidores y permite al segundo grupo conocer y elegir los productos que formarán parte de su dieta. En este sentido, el presente estudio tuvo como objetivo evaluar las etiquetas de diferentes marcas de margarinas vendidas

\footnotetext{
${ }^{1}$ Mestranda em Biometria e Estatística Aplicada, bolsista CAPES, UFRPE-Recife, beatrizcaren310@gmail.com ${ }^{2}$ Mestranda em Biometria e Estatística Aplicada, bolsista CNPq, UFRPE-Recife, andreanenildaagronomia@gmail.com

${ }^{3}$ Graduando em Agronomia, IFPE Campus Vitória, msouza04@gmail.com

${ }^{4}$ Graduando em Agronomia, UFRPE- Recife, adalbertofrancisco75@gmail.com

${ }^{5}$ Docente, UFRPE-Recife, moacyr2006@gmail.com
} 
en la ciudad de Recife-PE y compararlas con la legislación. Se recogieron al azar once marcas de margarina y se analizaron sus etiquetas. Para la identificación de los envases evaluados, se asignaron letras consecutivas de forma arbitraria. Los análisis se realizaron de acuerdo con las informaciones establecidas por las RDC n n $^{\circ} 59 / 03$ (BRASIL, 2003a), 360/03 (BRASIL, 2003b) y 259/02 (BRASIL, 2002). Los resultados mostraron que, a excepción de las marcas A, D y K, el $100 \%$ de las marcas en todos los atributos están dentro de los parámetros establecidos por la legislación brasileña a través de la Resolución RDC no 259 del 20 de septiembre de 2002 (BRASIL, 2002). Sin embargo, las tres marcas mencionadas no presentaban información en sus etiquetas sobre la ausencia de gluten. En cuanto a la información nutricional, según la Resolución no 359 y 360 de 2003, a excepción de las marcas B, H e I, en cuanto al contenido de fibra alimentaria, el $100 \%$ de las marcas estudiadas presentan la información exigida por la legislación. En cuanto a los contenidos de calcio, hierro y colesterol, el $100 \%$ de las muestras no los presentaban, respectivamente. Concluimos que se identificaron irregularidades en los criterios no nutricionales, debido a que algunas de las marcas no presentan información sobre la ausencia de gluten.

Palabras Clave: idoneidad, comunicación, información, verificación.

\begin{abstract}
Food labeling grants communication between enterprises and consumers and enables the latter group to know and choose the products that will be part of their diet. In this sense, the present study aimed to evaluate labels of different brands of margarine, commercialized in the city of Recife-PE, and compare them with the legislation. Eleven brands of margarine were randomly collected and their labels were analyzed. For the identification of the packages evaluated, consecutive letters were assigned arbitrarily. The analyses were performed according to the information established by RDC $\mathrm{n}^{\circ} 359 / 03$ (BRASIL, 2003a), 360/03 (BRASIL, 2003b) and 259/02 (BRASIL, 2002). The results showed that except for brands A, D and K, $100 \%$ of the brands in all attributes are within the parameters established by Brazilian legislation through Resolution RDC No. 259 of September 20, 2002 (BRASIL, 2002). However, the 3 brands mentioned above did not present information on their labeling regarding the absence of gluten. About the nutritional information, according to the Resolution $\mathrm{n}^{\mathbf{o}} 359$ and 360 of 2003, with the exception of the brands B, H and I, regarding the content of dietary fiber, $100 \%$ of the studied brands present the information required by the legislation. Regarding the calcium, iron and cholesterol contents, $100 \%$ of the samples did not present the contents, respectively. It can be concluded that irregularities were identified in the non nutritional criteria, due to some of the brands not presenting information on the absence of gluten.
\end{abstract}

Keywords: suitability, communication, information, verification.

\title{
INTRODUÇÃO
}

Os alimentos ultraprocessados são formulações industriais de substâncias derivadas de alimentos que contêm pouco ou nenhum alimento inteiro e constantemente são adicionadas de aromatizantes, corantes, emulsificantes e outros aditivos com função cosmética. (MONTEIRO et al., 2018) e (MONTEIRO et al., 2019).

No Brasil, a venda de alimentos ultraprocessados representou a $448 \mathrm{kcal}$ per capita/dia em 2014, valor 10,4\% superior ao registrado em 2009 (PAN AMERICAN HEALTH ORGANIZATION, 2019).

A margarina é uma emulsão de água e óleo que teve origem no país da França no ano de 1869 (RODRIGUES, 2004). O produto foi desenvolvido pelo químico francês MégeMouries apos uma solicitação do imperador por um produto que substituísse a manteiga (O`BRIEN, 2009). A invenção se destacou e houve produção em grande escala industrial e 
atualmente ainda é um produto relevante nas mesas dos consumidores brasileiros devido seu baixo custo e boas características sensoriais.

O interesse da população em relação aos alimentos que consomem aumenta significativamente, assim como crescem as pesquisas em relação as vantagens e aos prejuízos que os alimentos oferecem aos consumidores, confirmando que a saúde está diretamente relacionada à alimentação. Uma das principais causas de aflição dos consumidores com a alimentação está associada ao aumento dos casos de alergias e intolerâncias alimentares analisadas nos últimos anos na humanidade (SALEH, 2015; SOLÉ et al,. 2018).

O princípio fundamental que define as proclamações ou alegações de saúde nos alimentos é que devem provar-se cientificamente. Provavelmente, a definição mais acertada de “alegação" é a estabelecida em 1991 pelo Codex Alimentarius (Codex Alimentarius, 1991): "Qualquer representação que diz, sugere ou implica que um alimento tem certas características relacionadas com a sua origem, propriedades nutricionais, natureza, produção, processamento, composição ou qualquer outra qualidade". Todavia, o termo "alegação de saúde" é compreendido de diferentes formas segundo o país.

A rotulagem de alimentos concedem a comunicação entre empreendimentos e consumidores e possibilita, ao segundo grupo, conhecer e eleger os produtos que farão parte da sua alimentação. Neste contexto, destaca-se que, por muitas vezes, os rótulos das mercadorias alimentícias omitem algumas informações ou não a expõe de maneira clara e correta, conforme estudos apresentados por Miyoshi et al,. (2016) e Torquato, Occhioni e Souza (2016).

Neste sentido, o presente estudo teve o intuito de avaliar rótulos de diferentes marcas de margarinas comercializadas na cidade do Recife-PE e confrontando-os com as legislações.

\section{FUNDAMENTAÇÃO TEÓRICA}

\section{Definição, consumo e processo de fabricação das margarinas}

De acordo com a portaria n 372 de 04/09/1997 (BRASIL, 1997), margarina é definido como um produto gorduroso em emulsão estável com leite ou seus constituintes ou derivados, e outros ingredientes, destinados à alimentação humana com cheiro e sabor característico, contento $95 \%$ de lipídios.

Em estudo realizado por (COSTA et al., 2021) analisaram a frequência de consumo de alimentos ultraprocessados em entrevistados de população adulta das 27 capitais do Brasil. Os resultados demonstraram que as maiores frequências de consumo foram: margarina $(42,6 \%)$; pão de forma, de cachorro-quente ou de hambúrguer (32,8\%); refrigerante (27,7\%); salsicha, linguiça, mortadela ou presunto $(26,5 \%)$; chocolate, sorvete, gelatina, flan ou outra sobremesa 


\section{ANÁLISE DA ADEQUAÇÃO DOS RÓTULOS DE MARGARINAS}

industrializada (25,6\%); salgadinho de pacote ou biscoito/bolacha salgado (23,9\%); e biscoito/bolacha doce, biscoito recheado ou bolinho de pacote $(21,3 \%)$ (Figura 01$)$.

Figura 01: Frequência (\%) de consumo no dia anterior de grupos selecionados de alimentos ultraprocessados. População adulta ( $\geq 18$ anos) das capitais das 27 unidades federativas do Brasil,

$$
2019(n=52.443) \text {. }
$$

\begin{tabular}{lc}
\hline Grupos de alimentos ultraprocessados & $\%($ IC95\%) \\
\hline Margarina & $42,6(41,7-43,6)$ \\
\hline Pão de forma, de cachorro-quente ou de hambúrguer & $32,8(31,9-33,7)$ \\
Refrigerante & $27,7(26,8-28,6)$ \\
Salsicha, linguiça, mortadela ou presunto & $26,5(25,6-27,4)$ \\
Chocolate, sorvete, gelatina, flan ou outra sobremesa industrializada & $25,6(24,8-26,5)$ \\
Salgadinho de pacote (ou chips) ou biscoito/bolacha salgado & $23,9(23,1-24,7)$ \\
Biscoito/bolacha doce, biscoito recheado ou bolinho de pacote & $21,3(20,5-22,1)$ \\
Maionese, ketchup ou mostarda & $16,9(16,1-17,6)$ \\
logurte com sabor & $15,6(14,9-16,3)$ \\
Suco de fruta em caixa, caixinha ou lata & $15,0(14,3-15,8)$ \\
Refresco em pó & $12,8(12,0-13,5)$ \\
Bebida achocolatada & $11,9(11,2-12,6)$ \\
Macarrão instantâneo, sopa de pacote, lasanha congelada ou outro prato pronto & $6,6(6,1-7,2)$ \\
\hline
\end{tabular}

IC95\%: intervalo de confiança de 95\%.

Fonte: COSTA et al., (2021)

Em contrapartida a manteiga é um outro produto, onde é valorizada por seu sabor e aroma, sendo amplamente utilizada como fonte de gordura com a finalidade de enaltecer características sensoriais das formulações alimentícias (VIDANAGAMAGE, PATHIRAJE \& PERERA, 2016). Sua formulação é uma emulsão láctea água em óleo, obtida pela batedura do creme oriundo do desnate prévio do leite, contendo no mínimo de $80 \%$ de gorduras e no máximo $16 \%$ de água (BRASIL, 1996).

O procedimento de fabricação de margarina, pode ser dividido nas seguintes etapas: preparação da fase aquosa e fase lipídica, emulsificação, pasteurização, resfriamento, cristalização e amassamento, refusão, enchimento e embalagem (SPX, 2012). Em relação aos indicadores de processo, estes devem ser definidos para um nível apropriado de conteúdo de sólidos e da taxa de cristalização da gordura utilizada na formulação (CHRYSAN, 2005).

Os consumidores estão se tornando cada vez mais cientes da associação entre dieta e saúde. Assim, a demanda por uma dieta balanceada e alimentos funcionais que confirmam benefícios específicos à saúde é cada vez maior. Produtos alimentares benéficos podem ser 
caracterizados por vários atributos: baixo a moderado teor de sódio, açúcar e gorduras e densidade calórica significativamente reduzida, em comparação com os seus homólogos convencionais (PALZER, 2009).

\section{Rotulagem}

Rotulagem é toda inscrição, legenda, imagem ou toda matéria descritiva ou gráfica, escrita, impressa, estampada, gravada, gravada em relevo, litografada ou colada sobre a embalagem do alimento (BRASIL, 2002a).

Segundo Garcia e Carvalho (2011), o rótulo deve apresentar informações claras, precisas e ostensivas que não ofereça risco ao consumidor, principalmente àqueles com problemas de doenças crônicas como por exemplo a hipertensão e o diabetes mellitus. Já Cavada et al.,(2012), destacam que o rótulo tem a função de orientar os consumidores sobre a formulação dos elementos presentes no alimento, promovendo uma escolha por uma alimentação saudável e consciente.

Pesquisadores especializados na abordagem de fatores que interferem as escolhas de compra dos consumidores resultam em classifica-los em três categorias, sendo elas: influências ambientais, diferenças individuais e processos psicológicos de acordo com a (SUGANO; ABRANTES; SETTE 1999).

Em relação às diferenças individuais, estudos sugerem que a idade tem um significante impacto negativo sobre o entendimento da informação nutricional. Entretanto, o consumidor idoso, pesquisas sugerem que, em face do declínio cognitivo e da habilidade de percepção, este apresenta uma dificuldade adicional em utilizar a informação, particularmente quando sua apresentação é desorganizada e complexa (COLE, GAETH,1990).

Em contrapartida, Petrucelli (1996) argumenta que não é necessário o conhecimento de todas as variáveis. A inexistência da informação é suficiente para permitir a comparação entre produtos, afetar a escolha e aumentar a confiança do consumidor em suas decisões de compra.

Para Eves et al., (1994) sugerem que o interesse em rótulos é maior para as mulheres jovens, o que nem sempre vai de acordo com o conhecimento da informação quantitativa. Na prática, as mulheres fazem as suas escolhas com base na descrição qualitativa, pelo menos nos produtos estudados.

De acordo com Benhrens, Roing e Silva (2000), a preocupação com o bem-estar físico tem levado homens e mulheres a praticar exercícios e consumir uma alimentação mais balanceada, rica em fibras, vitaminas e minerais e com menos gorduras. Entretanto, mesmo com o interesse do consumidor em ler as informações contidas nos rótulos, é necessário saber 
quais são as procuradas com frequências e suas interpretações. Estas informações, devem ser claras e precisas, para garantir ao consumidor a escolha de seus alimentos de forma consciente.

A rotulagem dos alimentos embalados é obrigatória e regulamentada pela legislação brasileira por órgãos como o Ministério da Saúde, o Ministério da Agricultura, a Agência Nacional de Vigilância Sanitária (ANVISA), Pecuária e Abastecimento (MAPA) e pelo Instituto Nacional de Metrologia, Normalização e Qualidade Industrial (INMETRO) (TORQUATO et al., 2016). O governo brasileiro, em 1996, publicou a resolução número 36, na qual regulamenta a padronização da rotulagem de alimentos embalados.

Em 2002, a Agência Nacional de Vigilância Nacional Sanitária publicou a RDC 259 de 20/09/2002, que de acordo com essa portaria, tornaram-se obrigatórios: a apresentação da denominação de venda do alimento, a lista de ingredientes, a identificação da origem, o conteúdo líquido, a identificação do lote, a data de validade e as instruções sobre o preparo e uso do alimento, quando apropriado (BRASIL, 2002; LIMA et al., 2003).

É considerado rótulo toda inscrição que estiver apresentada na embalagem de um alimento, seja ele legenda, imagem, matéria descritiva ou gráfica, que esteja escrita, impressa, estampada, gravada, gravada em relevo, litografada ou colada sobre a embalagem do alimento (BRASIL, 2002). O alerta dos rotúlos exigidos pela ANVISA, deve-se a uma doença chamada Doença Celíaca, uma alergia instestinal ao glúten, qu se caracteriza a perda de peso, anemia, dores de cabeça, dentre outros sintomas.

Em 2005, a ANVISA publicou o Manual de Orientação às Indústrias de Alimentos, na qual foi regulamentada a obrigatoriedade de informações como: valor energético, teor de carboidratos, proteínas, gorduras totais, gorduras saturadas, gorduras trans, fibras, sódio, outros minerais, vitaminas e ainda a porcentagem de valores diários de referência com base em uma dieta de $2.000 \mathrm{Kcal}$ ou $8.400 \mathrm{KJ}$ (ANVISA, 2005). Faz necessário destacar que a informação de ferro, cálcio e colesterol, por sua vez, tornou-se facultativa desde dezembro de 2003 (BRASIL, 2003).

Contudo, é relevante ressaltar que os teores de ferro, cálcio e colesterol, que já constam nos rótulos de muitos alimentos, são informações úteis tanto para os consumidores quanto para os profissionais da área de saúde, e que podem deixar de existir. Mesmo que, haja parte da população brasileira que ainda não tenha adquirido o hábito de ler o rótulo de alimentos, outra parte, preocupada com o bem - estar e a saúde, proporcionados por uma alimentação adequada, já está acostumada a encontrar informações sobre o conteúdo e o valor diário de ferro, cálcio e colesterol. Esses dados, referentes aos teores desses nutrientes e que compõem o banco de dados analíticos de muitos fabricantes, por não mais serem obrigatórios, serão perdidos, com evidente 
prejuízo para a população, representando um verdadeiro retrocesso na evolução da legislação (FERREIRA e LANFER-MARQUEZ, 2007).

A normatização de rotulagem no Brasil foi uma grande vitória para o consumidor, no entanto, estudos têm apontado falhas na legislação, principalmente no que tange à informação nutricional (CÂMARA et al., 2008).e (LOMABNCO et al., 2009).

Ademais, se observa que, no que concerne às porções e medidas caseiras, o Brasil não dispõe de regulamentações de padrões de utensílios domésticos, os quais apresentam ampla variação de aptidão volumétrica, o que pode comprometer propósitos enunciados nesses documentos, principalmente os relacionados à ingestão de nutrientes (BOTELHO, 2007).

\section{METODOLOGIA}

A verificação dos rótulos de margarinas foi realizada a partir de amostras identificadas em diferentes supermercados da cidade do Recife-PE localizada na mesorreigão metropolitana do Recife.

Foram coletadas 11 marcas de margarinas aleatoriamente e realizada a análise de suas rotulagens. A metodologia utilizada foi a proposta por (ALBUQUERQUE et al., 2017), em estudo com creme de leite na cidade de Garanhuns-PE que se deu conforme descrita a seguir.

Para a identificação das embalagens avaliadas, foram atribuídas letras consecutivas e arbitrariamente, em ordem alfabetica de A a K. As análises foram realizadas de acordo com as informações estabelecidas pelas RDC n 359/03 (BRASIL, 2003a), 360/03 (BRASIL, 2003b) e 259/02 (BRASIL, 2002), que dispõem sobre a obrigatoriedade da rotulagem nutricional em alimentos produzidos e comercializados na ausência do cliente. Em relação aos parâmetros avaliados, levaram-se em consideração 11 critérios obrigatórios e 12 critérios pertinentes a informação nutricional metodologia também utilizada por Aquino et al (2017).

Os critérios obrigatórios considerados são: Lista de Ingredientes, Medida Caseira, Identificação da Origem, Denominação de Venda do Alimento, Conteúdo Líquido, Identificação do Lote, Data de Fabricação, Prazo de Validade, Conservação do Produto, Ausência de Glúten; Nome do País de Origem. Foram marcados com "X" os critérios que estavam de acordo e com "_" os que não estavam ou não foram apresentados. Os critérios referentes à rotulagem nutricional: Valor Energético (Kcal), Carboidratos (g), Porção (g), Sódio (mg), Cálcio (mg), Proteínas (g), Gorduras Totais (g), Gorduras Saturadas (g), Gorduras Trans (g), Ferro, Colesterol (mg), Fibra Alimentar (g).

Vale ressaltar que foram utilizadas apenas margarinas, descartando as manteigas, pois sua formulação difere das manteigas. 


\section{RESULTADOS E DISCUSSÃO}

Os resultados obtidos no estudo que se referem as informações de critérios obrigatórios não nutricionais podem ser visualizados na (Tabela 01) cujos atributos foram: lista de ingredientes, medida caseira, identificação da origem, denominação de venda do alimento, conteúdo líquido, identificação do lote, data de fabricação, prazo de validade, conservação do produto, ausência de glúten e nome do país de origem.

Tabela 01: Informações obrigatórias nos rótulos das margarinas comercializados em Recife-PE.

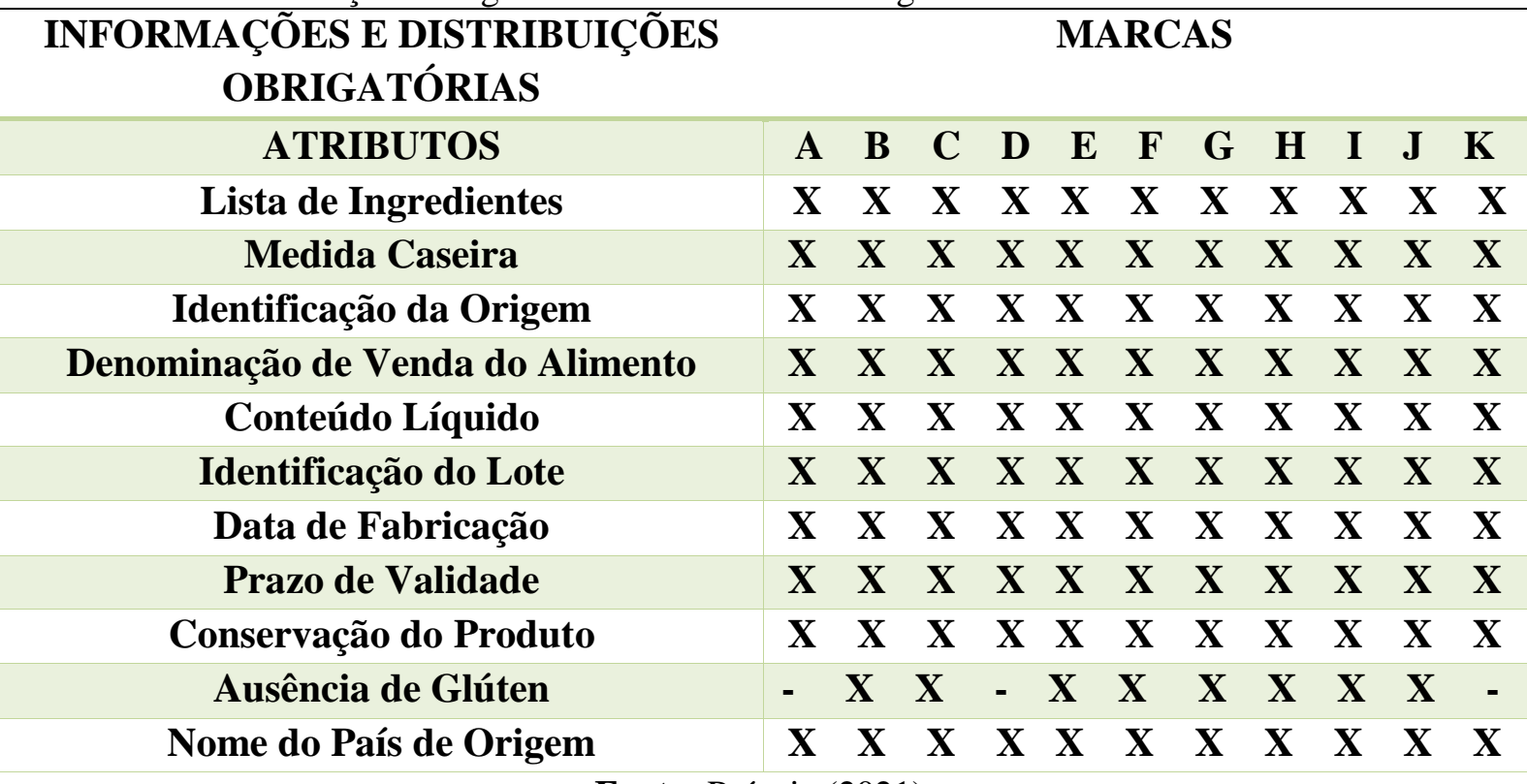

Fonte: Própria (2021).

Os resultados puderam demonstrar que exceto as marca A, D e K, 100\% das marcas em todos os atributos estão dentro dos parâmetros estabelecidos pela legislação brasileira através da Resolução RDC n 259 de 20 de setembro de 2002 (BRASIL, 2002). Porém as 3 marcas citadas acima não apresentaram informações em sua rotulagem quanto a ausência de glúten, o que torna alarmante, pois pode ocasionar consequências aos consumidores em relação a este componente.

Esta informação é extremamente relevante para o consumidor, expressa em caracteres de destaque no rótulo de alimentos. Assim, duas legislações se complementam para este fim, a Lei $n^{\circ}$ 8.543/92 (BRASIL, 1992), que estabelece a obrigatoriedade de impressão da advertência: "NÃO CONTÉM GLÚTEN" ou "CONTÉM GLÚTEN" em rótulos e embalagens de alimentos industrializados para comercialização. E posteriormente a Lei $n^{\circ}$ 10.674/03 (BRASIL, 2003c), obrigando os produtos alimentícios comercializados a trazerem 
na rotulagem a informação quanto à presença de glúten.

Segundo Rubio-Tapiaet et al., (2013), definem a doença celíaca como uma doença crônica de reação imunológica associada à ingestão de glúten, uma proteína encontrada no trigo, cevada e centeio que afeta principalmente o intestino delgado de pessoas com uma predisposição genética, obrigando-as a excluir o glúten de sua dieta.

A seguir na (Tabela 02) estão alocadas as informações nutricionais, segundo a Resolução no 359 e 360 de 2003 que com exceção da marca B, H e I no que diz respeito ao teor de fibra alimentar, $100 \%$ das marcas estudadas apresentam as informações exigidas pela legislação.

No que diz respeito aos teores de cálcio, ferro e colesterol identificou-se respectivamente valores de $100 \%$ das amostras não apresentam os teores. Isso pode ser explicado devido a não obrigatoriedade da expressão dos teores de colesterol, cálcio e ferro nos rótulos.

Tabela 02: Informações nutricionais nos rótulos das margarinas comercializados em Recife-PE.

\begin{tabular}{|c|c|c|c|c|c|c|c|c|c|c|}
\hline \multirow{2}{*}{$\begin{array}{c}\text { INFORMAÇÃO NUTRICIONAL } \\
\text { ATRIBUTOS }\end{array}$} & \multicolumn{10}{|c|}{ MARCAS } \\
\hline & $\mathbf{A}$ & B & $\mathbf{C}$ & D & $\mathbf{E} \mathbf{F}$ & $\mathbf{G}$ & $\mathbf{H}$ & I & $\mathbf{J}$ & $\mathbf{K}$ \\
\hline Valor Energético (Kcal) & $\mathbf{X}$ & $\mathbf{X}$ & $\mathbf{X}$ & $\mathbf{X}$ & $\mathbf{X} \mathbf{X}$ & $\mathbf{X}$ & $\mathbf{X}$ & $\mathbf{X}$ & $\mathbf{X}$ & $\mathbf{X}$ \\
\hline Carboidratos (g) & $\mathbf{X}$ & $\mathbf{X}$ & $\mathbf{X}$ & $\mathbf{X}$ & $\mathbf{X} \mathbf{X}$ & $\mathbf{X}$ & $\mathbf{X}$ & $\mathbf{X}$ & $\mathbf{X}$ & $\mathbf{X}$ \\
\hline Porção (g) & $\mathbf{X}$ & $\mathbf{X}$ & $\mathbf{X}$ & $\mathbf{X}$ & $\mathbf{X} \mathbf{X}$ & $\mathbf{X}$ & $\mathbf{X}$ & $\mathbf{X}$ & $\mathbf{X}$ & $\mathbf{X}$ \\
\hline Sódio (mg) & $\mathbf{X}$ & $\mathbf{X}$ & $\mathbf{X}$ & $\mathbf{X}$ & $\mathbf{X} \mathbf{X}$ & $\mathbf{X}$ & $\mathbf{X}$ & $\mathbf{X}$ & $\mathbf{X}$ & $\mathbf{X}$ \\
\hline Cálcio (mg) & - & - & - & - & - & - & - & - & - & - \\
\hline Proteínas (g) & $\mathbf{X}$ & $\mathbf{X}$ & $\mathbf{X}$ & $\mathbf{X}$ & $\mathbf{X} \mathbf{X}$ & $\mathbf{X}$ & $\mathbf{X}$ & $\mathbf{X}$ & $\mathbf{X}$ & $\mathbf{X}$ \\
\hline Gorduras Totais (g) & $\mathbf{X}$ & $\mathbf{X}$ & $\mathbf{X}$ & $\mathbf{X}$ & $\mathbf{X} \mathbf{X}$ & $\mathbf{X}$ & $\mathbf{X}$ & $\mathbf{X}$ & $\mathbf{X}$ & $\mathbf{X}$ \\
\hline Gorduras Saturadas (g) & $\mathbf{X}$ & $\mathbf{X}$ & $\mathbf{X}$ & $\mathbf{X}$ & $\mathbf{X} \mathbf{X}$ & $\mathbf{X}$ & $\mathbf{X}$ & $\mathbf{X}$ & $\mathbf{X}$ & $\mathbf{X}$ \\
\hline Gorduras Trans (g) & $\mathbf{X}$ & $\mathbf{X}$ & $\mathbf{X}$ & $\mathbf{X}$ & $\mathbf{X} \mathbf{X}$ & $\mathbf{X}$ & $\mathbf{X}$ & $\mathbf{X}$ & $\mathbf{X}$ & $\mathbf{X}$ \\
\hline Ferro & - & - & - & - & - & - & - & - & - & - \\
\hline Colesterol (mg) & - & - & - & - & - & - & - & - & - & - \\
\hline Fibra Alimentar (g) & $\mathbf{X}$ & - & $\mathbf{X}$ & $\mathbf{X}$ & $\mathbf{X} \mathbf{X}$ & $\mathbf{X}$ & $\mathbf{X}$ & - - & $\mathbf{X}$ & $\mathbf{X}$ \\
\hline
\end{tabular}

A RDC $\mathrm{n}^{\circ}$ 360/03 (BRASIL, 2003c) estabelece que "INFORMAÇÃO NUTRICIONAL" deve estar em local de destaque com relação as demais informações prestados na rotulagem nutricional. Ainda estabelece que para forma reduzida, é permitida a sua utilização desde que quantidade por porção do nutriente não seja significativa, sendo assim, substituído pela expressão: "não contém quantidade significativa de: nome do nutriente".

Outra Legislação importante relacionada a esta temática trata-se da RDC no 359/03 
(BRASIL, 2003b), que estabelece o regulamento técnico de porções de alimentos embalados para fins de rotulagem nutricional. Essa regulamentação define a quantidade média do alimento a ser consumido por pessoas saudáveis, maiores de 36 meses de idade, em cada ocasião de consumo levando em consideração a promoção de uma alimentação saudável e segura. E ainda, descreve a medida caseira como aquele utensílio costumeiramente utilizado pelo consumidor para medir os alimentos, já unidade refere-se a cada produto alimentício igual ou similar, contido em uma mesma embalagem e a fração como a parte de um todo.

A (Figura 02) a seguir, corrobora com os resultados encontrados no rótulo da marca $\mathrm{C}$ de margarina utilizada neste estudo, em relação aos critérios nutricionais.

Figura 02: Informação nutricional presente no rótulo da marca $\mathrm{C}$ de margarina.

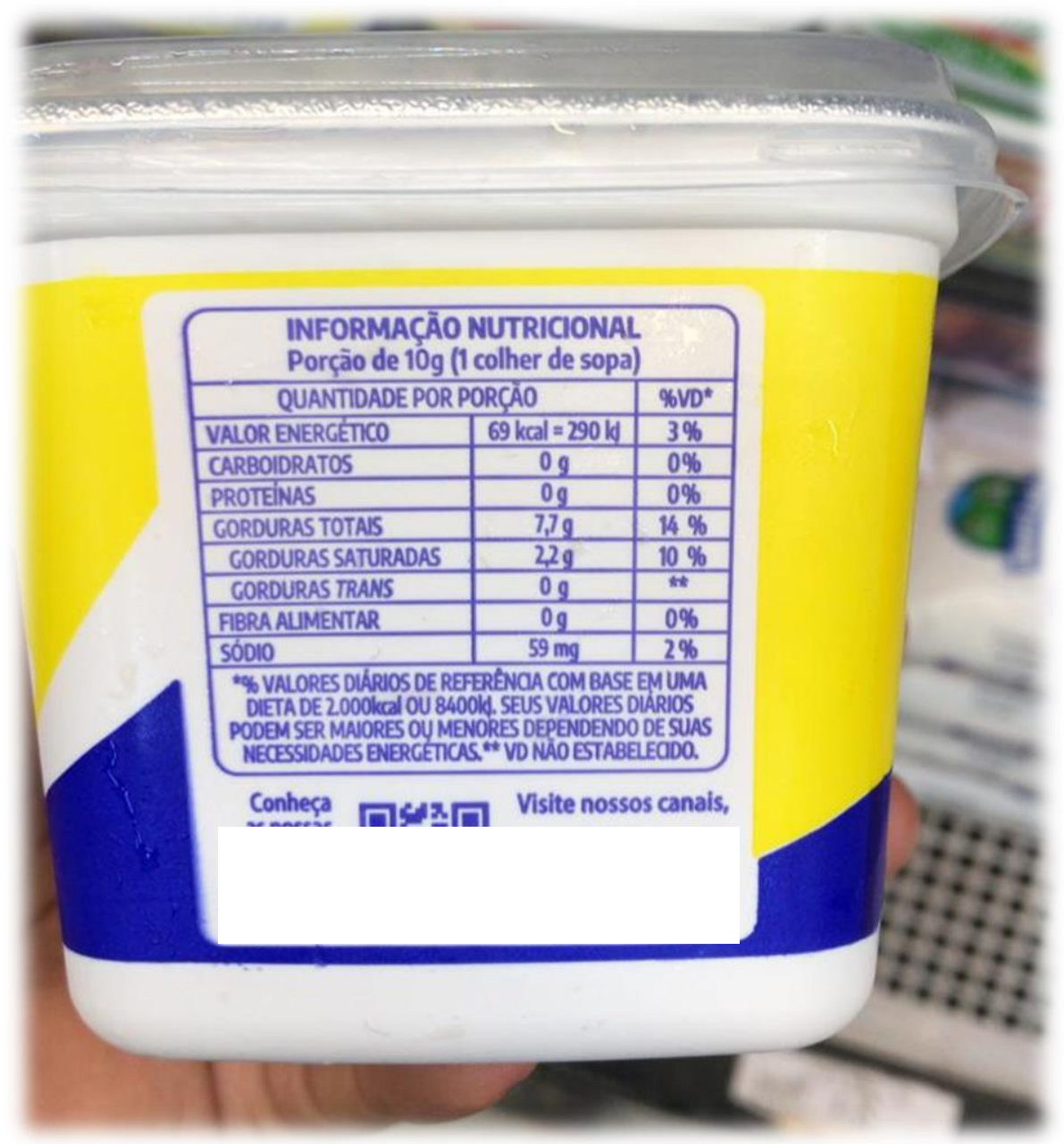

Fonte: Própria (2021).

O registro reforça a presença das informações tais como: valor energético, carboidratos, porção, sódio, proteínas, gorduras totais, gorduras saturadas, gorduras trans e fibra alimentar e a não presença do cálcio, ferro e colesterol, tendo em vista que não são critérios obrigatórios conforme discutido anteriormente. 


\section{CONCLUSÕES}

Conclui-se que foi identificado irregularidades nos critérios não nutricionais, devido algumas marcas (B, H , I) não apresentarem a informação sob a ausência de glúten, tornandose preocupante devido as consequências do consumo deste componente.

Desta forma, se faz necessário uma maior fiscalização dos órgãos competentes e novos estudos referentes a outros produtos oriundos animal e/ou vegetal afim de identificar outros alimentos industrializados, com base nesse e em outros resultados encontrados e posteriormente a realização da divulgação pela comunidade científica.

\section{REFERÊNCIAS}

AGÊNCIA NACIONAL DE VIGILÂNCIA SANITÁRIA - ANVISA, 2016 Guia 05/2016 2a Versão. Disponível em: https://www.saude.rj.gov.br/comum/code/ MostrarArquivo.php?C=MzIxOQ\%2C\%2C. Acesso em: 17 mai 2018. 2.

AGÊNCIA NACIONAL DE VIGILÂNCIA SANITÁRIA - ANVISA; UNIVERSIDADE DE BRASÍLIA - UnB. Rotulagem Nutricional Obrigatória: Manual de Orientação às Indústrias de Alimentos. 2. versão. Brasília: ANVISA, UnB, 2005. 44 p.

ALBUQUERQUE, K. G. S.; XAVIER, L. C. A.; CHINELATE, G. C. B. Avaliação de Rótulos de Cremes de Leite Comercializados no Município de Garanhuns - Pe. II CONGRESSO INTERNACIONAL DAS CIÊNCIAS AGRARIAS. COINTER - PDVAgro. 2017.

AQUINO, C. E. F.; MORAIS, M. C. G.; OLIVEIRA, R. M. S.; ALMEIDA, F. L. C.; OLIVEIRA, E.N. A.; OLIVEIRA, S. N. Avaliação da rotulagem de cremes de leite comercializados na cidade de Pau dos Ferros-RN. Revista Brasileira de Agrotecnologia, Brasil, v. 7, n. 1, 2017.

BENHERNS,J.H.;ROIG,S.M.;SILVA,M.A.P.da.Aspectos de funcionalidade, de rotulagem e de aceitação de extrato hidrossolúvel de soja fermentado e culturas lácteas probióticas. Boletim da Sociedade Brasileira de Ciência e Tecnologia de Alimentos, Campinas,v.34,n.2,p.61128,jul./dez.2000.

BOTELHO RA. Características dos utensílios alimentícios no Brasil e seu impacto na construção de tabelas de pesos e medidas caseiras. Nutr Brasil. 2007;6(2):105-110.

BRASIL. Ministério da Saúde. Agência Nacional de Vigilância Sanitária (ANVISA). Decreto Lei ${ }^{\circ}$ 986, 21 de outubro de 1969. Institui Normas Básicas Sobre Alimentos. Brasília, 21 de out. 1969.

BRASIL. Lei $\mathrm{n}^{\circ}$ 8.543, de 23 de dezembro de 1992. Determina a impressão de Advertência em Rótulos e embalagens de alimentos industrializados que contenham Glúten. Agência Nacional de Vigilância Sanitária (ANVISA),, Disponível em: http://www.anvisa.gov.br/ Acessado em: 10 out. 2021. 
BRASIL. Portaria $\mathrm{n}^{\circ}$ 146, de 07 de março de 1996. Dispõe sobre o Regulamento Técnico de Identidade e Qualidade dos Produtos Lácteos. Brasília: Ministério da Agricultura, Pecuária e Abastecimento. 1996.

BRASIL. Ministério da Agricultura, Pecuária e Abastecimento. Portaria $\mathrm{n}^{\circ}$ 372, de 4 de setembro de 1997. Regulamento técnico para fixação de identidade e qualidade da margarina. Diário Oficial da União, 1997.

BRASIL. Ministério da Saúde. Agência Nacional de Vigilância Sanitária (ANVISA). Decreto Lei $n^{\circ}$ 6437, 20 de agosto de 1977. Configura infrações à legislação sanitária federal, estabelece as sanções respectivas, e dá outras providências. Diário Oficial da União. Brasília, 20 ago. 1977. 8 .

BRASIL. Agência Nacional de Vigilância Sanitária. Resolução de Diretoria Colegiada n ${ }^{\circ} 259$, de 20 de setembro de 2002. Aprova o Regulamento Técnico sobre Rotulagem de Alimentos Embalados. Diário Oficial da União, Brasília, 23 set. 2002. 6.

BRASIL. Agência Nacional de Vigilância Sanitária. Resolução de Diretoria Colegiada n ${ }^{\circ}$ 344, de 13 de setembro de 2002. Aprova o Regulamento Técnico para a Fortificação das Farinhas de Trigo e das Farinhas de Milho com Ferro e Ácido Fólico. Diário Oficial Da União, Brasília, 18 de dez. 2002. 7.

BRASIL. Ministério da Saúde. Informação nutricional. Resolução RDC no 360, de 23 de dezembro de 2003. Diário Oficial da República Federativa do Brasil, Poder Executivo, Brasília, DF, 26 dez. 2003b. Seção 1. 5.

BRASIL. Lei $\mathrm{n}^{\circ} 10.674$, de 16 de maio de $2003 \mathrm{~d}$, obriga os produtos alimentícios comercializados a informar a presença de Glúten,como prevenção da doença celíaca. Disponível. ANVISA. em: http://www.anvisa.gov.br/Acessadoem: 09 out. 2021.

CÂMARA, M. C.C.; MARINHO, C. L. C.; GUILAM, C. M., BRAGA, A. M. C. B. A produção acadêmica sobre a rotulagem de alimentos no Brasil. Rev Panam Salud Publica. 2008; 23(1):52-58. http://dx.doi.org/10.1590/ S1020-49892008000100007.

CAVADA, G.S.; PAIVA, F.F.; HELBIG, E.; BORGES, L. R. Rotulagem nutricional: você sabe o que está comendo?Braz. J. Food Technol, Pelotas, RS, v1, n.1, p.84-88, Dez, 2012.

CHRYSAN, M. M. Margarines and Spreads. In: SHAHIDI, F. (Ed.). Bailey's Industrial Oil and Fat Products. 6. ed.: John Wiley \& Sons Inc., p. 33-82, 2005.

CODEX ALIMENTARIUS 1991. Second Edition. Codex Guide-lines on Claims. (CAC/GL 1-1979, rev 1-1991). Geneva. WHO.

COLLE, C.A.;GAETH, G.J. Cognitive and age-related diferences in the abilility to use nutrition information. Journal of Marketing Research, v.27, n.2, p. 175-184,1990.

COSTA, C. dos S.; SATTAMINI, I. F.; STEELE, E. M.; LOUZADA, M. L. da C.; CLARO, R. M.; MONTEIRO, C. A. Consumo de alimentos ultraprocessados e associação com fatores sociodemográficos na população adulta das 27 capitais brasileiras (2019). Revista de Saúde Pública, v. 55, n. 47, p. 1-9, 2021. 
EVES,A.et al. Influence of nutrition information on the altitudes and knowledge of dieters. Nutrition and Food Science,n.5,p.17-21,1994.

FERREIRA, A. B., LANFER-MARQUEZ, Ú. M. Legislação brasileira referente à rotulagem nutricional de alimentos. Rev. Nutr., v. 20, n. 1, p.83-93, 2007.

GARCIA, P.P.C.; CARVALHO, L.P.S. Análise da rotulagem nutricional de alimentos diet e light. Ensaios e Ciência: Ciências Biológicas, Agrárias e da Saúde, v.15, n.4, p.89-103, 2011

LOMABNCO MC, Vedovato MG, Cano CB, Bastos MHD. Fidedignidade de rótulos de alimentos comercializados no município de São Paulo. SP. Rev Saúde Pública. 2009;43(3):499-505. http://dx.doi. org/10.1590/S0034-89102009005000020.

MIYOSHI, L. Y; GALVÃO, J. A.; WEBER, L. F. S.; MANTOVANI, S. L.; JUNIOR, P. I. T.; RALDI,T. Rotulagem de leites UHT comercializados no varejo. Rev. Inst. Laticínios Cândido Tostes, Juiz de Fora, v. 71, n. 1, p. 19-25, jan/mar, 2016.

MONTEIRO CA, CANNON G, MOUBARAC JC, LEVY RB, LOUZADA MLC, JAIME PC. The UN Decade of Nutrition, the NOVA food classification and the trouble with ultraprocessing. Public Health Nutr. 2018;21(1):5-17. https://doi.org/10.1017/S1368980017000234.

MONTEIRO CA, CANNON G, LEVY RB, MOUBARAC JC, LOUZADA ML, RAUBER F, et al. Ultra-processed foods: what they are and how to identify them. Public Health Nutr. 2019;22(5):936-41. https://doi.org/10.1017/S1368980018003762.

O`BRIEN, R. D. Fat and oils: formulating and processing for applications. 3. Ed. Boca Raton: Crc Press, 2009. 766 p.

PALZER, S. Food structures for nutrition, health and wellness. Trends in Food Science \& Technology. v.20, n.5, p.194-200, 2009.

PAN AMERICAN HEALTH ORGANIZATION. Ultra-processed food and drink products in Latin America: sales, sources, nutrient profiles, and policy implications. Washington, DC: PAHO; Disponível em: https://iris.paho.org/handle/10665.2/51094. Acessado em: 19 out 2021.

RODRIGUES, J. N.; FILHO, J. M.; TORRES, R. P.\&GIOIELLI, L. A. Caracterização físicoquímica de creme vegetal enriquecido com ésteres de fitosteróis. Rev. Bras. Cienc. Farm. Braz. J. Pharm. Sci. vol. 40, n. 4, out./dez., 2004.

PETRUCELLI, P.J. Consumer na marketing implications of information provision: the case of the NLEA of 1990. Journal of Public Policy and marketing, v.15, n.1,p.150-153,1996.

RUBIO-TAPIA, A.; HILL, I. D.; KELLY, C. P.; CALDERWOOD, A. H.; MURRAY, J. A. American College of Gastroenterology. ACG clinical guidelines: diagnosis and management of celiac disease. Nature, Am J Gastroenterol. 2013, p. 656-671. Disponível em: https://www.nature.com/articles/ajg201379.pdf. Acessado em: 10 out 2021. 
SALEH, N. Alergia alimentar: casos aumentam quase $30 \%$ ao ano, aponta estudo americano. Revista Crescer, 2015. Disponível em: . Acesso em: 20 set. 2021.

SPX ® Corporation. Margarine Production - Technology and Process. Charlotte, North Carolina, jul. 2012. Disponível em: . Acessado em: 09/10/2021.

SOARES, N. R. et al. (2019). Estabilidade de manteigas enriquecidas com carotenoides de pimentão e abóbora. Revista Ingi -Indicação Geográfica e Inovação, 3 (4), 490-502.

SOLÉ, D; SILVA, L. R; COCCO, R. R; FERREIRA, C. T; SARNI, R. O; OLIVEIRA, L. C;. ASTORINO, A. C; WEFFORT, V; MORAIS, M. B; BARRETO, B. P; OLIVEIRA, J. C; CASTRO, A. P. M; FRANCO, J. M; CHONG NETO, H. J; ROSÁRIO, N. A; ALONSO, M. L. O; SARINHO, E. C; YANG, A; MARANHÃO, H; TOPOROVSKI, M. S; EPIFANIO, M; WANDALSEN, N. F; RUBINI, N. M. Consenso Brasileiro sobre Alergia Alimentar: 2018 Parte 1 - Etiopatogenia, clínica e diagnóstico. Documento conjunto elaborado pela Sociedade Brasileira de Pediatria e Associação Brasileira de Alergia e Imunologia. Arq Asma Alerg Imunol, v.2, n.1, 2018.

SUGANNO, J. Y.; ABRANTES, L.A.; SETTE , R.S. Perfil do consumidor de iogurte na Região Oeste Paulista, $1999 . \quad$ Disponível em: www.dae.ufla.br/biblioteca.4congABAR/arc1999\%5Carc8.pdf. Acesso em: 20 set. 2021.

TORQUATO, V. S. A; OCCHIONI, C. V. O; SOUZA, M. R. P. Avaliação da rotulagem de leites UAT comercializados por estabelecimentos varejistas no município do Rio de Janeiro RJ, Brasil. Vet. Not., Uberlândia, v.22, n. 2, p.19-23, 2016.

VIDANAGAMAGE, S. A.; PATHIRAJE, P. M. H. D.; PERERA, O. D. A. N. (2016). Effects of Cinnamon (Cinnamomum verum) extract on functional properties of butter. Procedia Food Science, 6, 136-142. 